\section{1,3-Dipolare Cycloadditionen der Knallsäure}

Von R. Huisgen und $M$. Christl $\left.{ }^{*}\right]$

Quilico et al. [1,2] beschrieben Cycloadditionen der Knallsäure (2), die aus Natriumfulminat mit 20-proz. Schwefelsäure oder aus Formhydroximsäure-jodid (1) mit Base freigesetzt wurde. Die rasche Polymerisation von (2) ist für die niedrigen Addukt-Ausbeuten verantwortlich. Beispielsweise wurden mit Acetylen nur 3\% Isoxazol, aber $20 \%$ des Isoxazol-3-aldoxims (Addukt der dimeren Knallsäure) erhalten [1]. Wir überführten Quecksilber-fulminat ohne Umweg über das Natriumsalz ${ }^{[3]} \mathrm{mit} \mathrm{HJ} / \mathrm{KJ}$ in $60 \%$ Ausb. in das reine kristalline Formhydroximsäure-jodid (1). Durch langsames Zutropfen von einem Äquivalent Triäthylamin zur eisgekühlten, gerührten Lösung von (I) in überschüssigem Dipolarophil, eventuell mit Äther verdünnt, setzt man die Knallsäure in Freiheit. Die niedrige Stationärkonzentration von (2) bietet für die Bildung von $\Delta^{2}$-Isoxazolinen oder Isoxazolen mit olefinischen bzw. acetylenischen Dipolarophilen optimale Bedingungen.

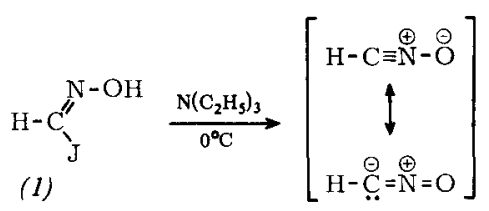

(2)

Methyl-acrylat liefert mit $90 \%$ Ausbeute $\Delta^{2}$-Isoxazolin5-carbonsäure-methylester(3), der durchDestillation imHochvakuum gereinigt wird $\left(\mathrm{Kp}=60-65^{\circ} \mathrm{C} / 0,001\right.$ Torr) [NMR $\left(\mathrm{CDCl}_{3}\right): \tau=2,72(\mathrm{H}-3, \mathrm{t}), \mathrm{J}_{34}=1,7 \mathrm{~Hz} ; \tau=6,67(\mathrm{H}-4, \mathrm{q})$, $\left.\mathrm{J}_{45}=9,1 \mathrm{~Hz} ; \tau=5,00(\mathrm{H}-5, \mathrm{t}) ; \tau=6,20\left(\mathrm{OCH}_{3}, \mathrm{~s}\right)\right]$. Uberschüssiges Triäthylamin überführt (3) quantitativ in 3-Cyan2-hydroxypropionsäure-methylester (4) [NMR $\left(\mathrm{CDCl}_{3}\right)$ : $\tau=5,48(\mathrm{H}-2, \mathrm{t}), \mathrm{J}_{23}=5,5 \mathrm{~Hz} ; \tau=6,12\left(\mathrm{OCH}_{3}, \mathrm{~s}\right) ; \tau=6,37$ (OH, s, breit); $\tau=7,12(\mathrm{H}-3, \mathrm{~d})$; IR (Film): $2248(\mathrm{C} \equiv \mathrm{N})$, $\left.3400 \mathrm{~cm}^{-1}(\mathrm{OH})\right]$.
(H-2, d), $\mathrm{J}_{23}=3,5 \mathrm{~Hz} ; \tau=6,81$ (H-3, Octuplett); $\tau=8,67$ $\left(4-\mathrm{CH}_{3}, \mathrm{~d}\right), \mathrm{J}_{34}=7,3 \mathrm{~Hz} ; \tau=6,11\left(\mathrm{OCH}_{3}, \mathrm{~s}\right) ; \tau=6,28(\mathrm{OH}, \mathrm{s}$, breit). IR (Film) : $2270(\mathrm{C} \equiv N), 3400 \mathrm{~cm}^{-1}(\mathrm{OH})$. Die erst bei $120-150^{\circ} \mathrm{C}(\mathrm{Bad}) / 0,001$ Torr übergehenden 2:1-Addukte (7) und (8) wurden im Gemisch NMR-analysiert.

Zimtsäure-methylester ergibt analog 1:1- und 2:1-Addukte (23 bzw. $33 \%$ Ausbeute). Die 1:1-Addukte bestehen im Verhältnis $76: 24$ aus den 4- und 5-Carbonsäureestern $(70: 30$ bei der Anlagerung von Benzonitril-oxid an Zimtsäureester ${ }^{[4]}$ ). Norbornen reagiert an seiner winkelgespannten Doppelbindung mit (2) in $76 \%$ Ausbeute $\mathrm{zu}(12), \mathrm{Kp}=55-60{ }^{\circ} \mathrm{C} /$ 0,001 Torr [NMR $\left(\mathrm{CDCl}_{3}\right): \tau=3,08(\mathrm{H}-3, \mathrm{~d}), \mathrm{J}_{33 \mathrm{a}}=$ $1 \mathrm{~Hz} ; \tau=6,85(\mathrm{H}-3 \mathrm{a}, \mathrm{q}), \mathrm{J}_{3 \mathrm{a} 7 \mathrm{a}}=8,2 \mathrm{~Hz} ; \tau=5,61(\mathrm{H}-7 \mathrm{a}$, d)]. Die fehlende Kopplung von $\mathrm{H}-3 \mathrm{a}$ und $\mathrm{H}-7 \mathrm{a}$ mit dem Brückenkopf-Wasserstoff zeigt die exo-Addition an. Erst 12-stündiges Kochen von (12) in Triäthylamin liefert das Nitril (13) mit $93 \%$ Ausbeute $\left[\mathrm{Fp}=35-38^{\circ} \mathrm{C}\right.$; IR: 2270 $\left.(\mathrm{C} \equiv \mathrm{N}), 3450 \mathrm{~cm}^{-1}(\mathrm{OH})\right]$.<smiles>C1CC2CC1CC21NOC2NC3CCC2C31</smiles>

(12)<smiles>N#CC1C(O)CC2CCCCC21</smiles>

(13)

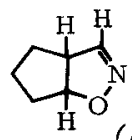

(14)
Cyclopenten und (2) ergeben (14) mit $8 \%$ Ausbeute $(\mathrm{Kp}=$ 95- $110{ }^{\circ} \mathrm{C}$ (Bad)/10 Torr). Das ebenfalls mit $8 \%$ Ausbeute isolierte Addukt (15) des Cyclooctatetraens leitet sich vom Bicyclo[4.2.0]octadien-Skelett ab, wie das Verhältnis (Vinyl-H) :(tert.H) zeigt.
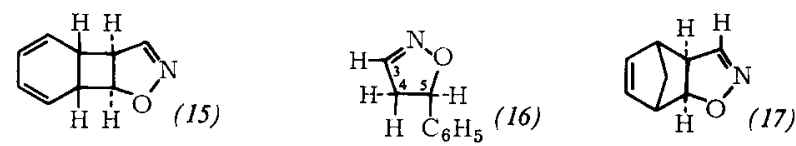

Aus der Cycloaddition von (2) an Styrol geht das 5-Phenyl$\Delta^{2}$-isoxazolin (16) $\left(\mathrm{Kp}=90-100^{\circ} \mathrm{C} / 0,001\right.$ Torr $)$ mit $70 \%$ Ausbeute hervor. Das NMR-Spektrum $\left(\mathrm{CDCl}_{3}\right)$ beweist die Struktur: $\tau=2,91(\mathrm{H}-3, \mathrm{t}), \mathrm{J}_{34}=1,7 \mathrm{~Hz}$; die $\mathrm{CH}_{2}$-Gruppe an C-4 bildet das erwartete 16-Linien-Signal $\left(J_{\text {gem }}=-17,6 \mathrm{~Hz}\right)$.

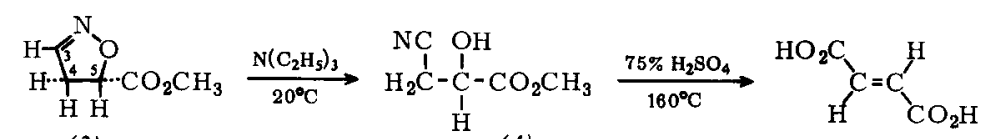

(3)

(4)

Aus Methyl-crotonat und (2) erhält man die Addukte (5) und (6) im Verhältnis 62:38 (Ausbeute $36 \%$ ) und die 2:1Addukte (7) und (8) im Verhältnis 45:55 (31\% Ausbeute). Der Cycloaddition von (2) zu (5) und (6) erwächst hier also schon eine Konkurrenz in der Dimerisierung von (2) zum. Oximinoacetonitril-oxid, das sich mit dem Dipolarophil zu (7) und (8) vereinigt. Benzonitril-oxid ergibt mit Methylcrotonat (9) und (10) im Verhältnis 34:66 [4].

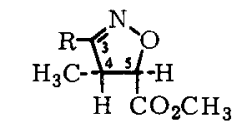

(5), $\mathrm{R}=\mathrm{H}$

(7), $\mathrm{R}=-\mathrm{CH}=\mathrm{NOH}$

(9), $\mathrm{R}=\mathrm{C}_{6} \mathrm{H}_{5}$

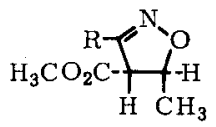

(6), $\mathrm{R}=\mathrm{H}$

(8), $\mathrm{R}=-\mathrm{CH}=\mathrm{NOH}$

(10), $\mathrm{R}=\mathrm{C}_{6} \mathrm{H}_{5}$<smiles>CC(=O)C(O)C(C)C#N</smiles>

Die Addukte (5) und (6) lassen sich dünnschichtchromatographisch (mit Benzol auf Kieselgel $\mathrm{PF}_{254}+366$ ) trennen. NMR-Spektren beweisen die Strukturen. Die durch Triäthylamin katalysierte Ringöffnung zu (11) läuft bei (5) schon in der Kälte ab. NMR-Spektrum von (11) $\left(\mathrm{CDCl}_{3}\right): \tau=5,74$
Erst in kochendem Triäthylamin findet Ringöffnung zum Hydroxynitril statt.

Das Norbornadien-Addukt (17), durch Destillation (Kp = $60-65^{\circ} \mathrm{C} / 0,001$ Torr) mit $41 \%$ Ausbeute isoliert und durch NMR- und IR-Spektren strukturell gesichert, erleidet bei $140-160^{\circ} \mathrm{C}$ eine Retro-Diels-Alder-Reaktion, die zu Cyclopentadien und Isoxazol ( $90 \%$ Ausbeute) führt. NMR-Spektrum von (18) $\left(\mathrm{CDCl}_{3}\right): \tau=1,66(\mathrm{H}-3, \mathrm{~d}), \tau=3,59(\mathrm{H}-4, \mathrm{t})$ und $\tau=1,49\left(\mathrm{H}-5\right.$, d) mit $\mathbf{J}_{34}=\mathrm{J}_{45}=1,5 \mathrm{~Hz}$.

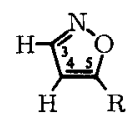

(18) $\mathrm{R}=\mathrm{H}$

(19). $\mathrm{R}=\mathrm{C}_{6} \mathrm{H}_{5}$

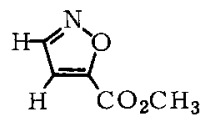

(20)

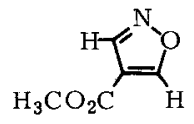

(21)
Die Umsetzung von (2) mit Phenylacetylen liefert mit 51\% Ausbeute das 5-Phenyl-isoxazol (19). NMR $\left(\mathrm{CDCl}_{3}\right): \tau=1,72$ $(\mathrm{H}-3, \mathrm{~d})$ und $\tau=3,52\left(\mathrm{H}-4\right.$, d) mit $\mathbf{J}_{34}=1,9 \mathrm{~Hz}$.

Methyl-propiolat lagert Knallsäure in beiden Richtungen an. Mit 50\% Ausbeute erhält man ein 84:16-Gemisch aus (20), $\mathrm{Fp}=47-50^{\circ} \mathrm{C}[5]$ und (21); NMR-Spektrum von (20) $\left(\mathrm{CDCl}_{3}\right): \tau=1,47$ und $2,93\left(\mathrm{H}-3\right.$ und $\mathrm{H}-4$, d) mit $\mathbf{J}_{34}=1,8$ $\mathrm{Hz}, \tau=5,98\left(\mathrm{OCH}_{3}, \mathrm{~s}\right)$; NMR-Spektrum von $(21)\left(\mathrm{CDCl}_{3}\right)$ : $\tau=1,30(\mathrm{H}-3, \mathrm{~s}), \tau=0,85(\mathrm{H}-5, \mathrm{~s})$ und $\tau=6,09\left(\mathrm{OCH}_{3}, \mathrm{~s}\right)$. 
Benzonitril-oxid reagiert mit Methyl-propiolat zum 5- und 4-Carbonsäureester im Verhältnis 72:28 [4],

Diese Cycloadditionen sind nur mit der kürzlich IR-spektroskopisch gesicherten [6] Formonitriloxid-Struktur (2) der Knallsäure vereinbar, nicht dagegen mit der klassischen Carboxim-Formel.

$$
\text { Eingegangen am 20. März } 1967
$$

[Z 478]

[*] Prof. Dr. R. Huisgen und Dipl.-Chem. M. Christl Institut für Organische Chemie der Universität 8 München 2, Karlstraße 23

[1] A. Quilico u. G. Stagno d'Alcontres, Gazz. chim. ital. 79, 654, 703 (1949).

[2] G. Stagno d'Alcontres u. G. Fenech, Gazz. chim. ital. 82, 175 (1952).

[3] A. Quilico u. L. Panizzi, Gazz. chim. ital. 72, 155 (1942).

[4] R. Sustmann, Dissertation, Universität München, 1965.

[5] Synthese auf anderem Wege: A. Quilico u. L. Panizzi, Gazz. chim. ital. $72,458(1942) ; \mathrm{Fp}=49-50^{\circ} \mathrm{C}$.

[6] W. Beck u. K. Feldl, Angew. Chem. 78, 746 (1966); Angew. Chem. internat. Edit. 5, 525 (1966).

\section{1,3-Cycloadditionen der Nitrone an Methylen-phosphorane}

\section{Von $J$.Wulff und R. Huisgen ${ }^{[*]}$}

Vor kurzem wurde gezeigt, daß Methylenphosphorane Dipolarophile sind und sich mit Nitriloxiden zu fünfgliedrigen Cycloaddukten vereinigen [1,2]. Als 1,3-Dipole, die in der Sextett-Grenzformel keine Doppelbindung mehr besitzen [3], haben wir die Azomethin-oxide (Nitrone) gewählt.

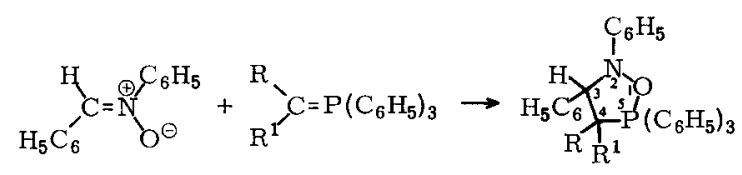$$
\text { (1) }
$$

(2)

(3)

$$
\begin{aligned}
& \text { (a), } \mathrm{R}=\mathrm{R}^{1}=\mathrm{H} \\
& \text { (b), } \mathrm{R}=\mathrm{R}^{1}=\mathrm{D} \\
& \text { (c), } \mathrm{R}=\mathrm{C}_{6} \mathrm{H}_{5}, \mathrm{R}^{1}=\mathrm{H} \\
& \text { (d), } \mathrm{R}=\mathrm{R}^{1}=\mathrm{CH}_{3}
\end{aligned}
$$

Setzt man $C, N$-Diphenylnitron (1) mit Methylen-triphenylphosphoran (2a) im Verhältnis $1: 1$ in Äther bei $20^{\circ} \mathrm{C}$ um, so scheidet sich das farblose kristalline 1,2,5-OxazaphospholDerivat ( $3 a$ ), Zers.-P. $136-137^{\circ} \mathrm{C}$, mit $93 \%$ Ausbeute aus; $\mathrm{C}-$, $\mathrm{H}-$ und $\mathrm{N}$-Analyse sowie die osmometrische Molgewichtsbestimmung ergaben für $(3 a)$ wie für die übrigen neuen Verbindungen korrekte Werte. NMR-Spektrum $\left(\mathrm{CDCl}_{3}\right)$ von (3a) : $\mathrm{H}-4$ und $\mathrm{H}-4^{\prime}$ als Octupletts bei $\tau=6,36$ und $6,93 \mathrm{mit}$ $\mathbf{J}_{44^{\prime}}=-16,3, \mathbf{J}_{34}=8,5, \mathrm{~J}_{45}=12,0, \mathbf{J}_{34^{\prime}}=7,0$ und $\mathrm{J}_{4^{\prime} 5}=12,4$ Hz. Die Ähnlichkeit der Koppelungen von $\mathrm{H}-3\left(\mathrm{~J}_{35}=8,1 \mathrm{~Hz}\right)$ hat ein Quadruplett bei $\tau=5,30$ zur Folge. Das ${ }^{31} \mathrm{P}$-Signal bei $+58,6 \mathrm{ppm}\left(\mathrm{CDCl}_{3}, 85\right.$-proz. Phosphorsäure als äußerer Standard) weist auf pentakovalenten Phosphor. Läge statt (3a) das isomere Phosphonium-Zwitterion (4) vor, so wäre eine große negative chemische Verschiebung zu erwarten [4].

Die Deutung des ${ }^{1} \mathrm{H}-\mathrm{NMR}-$ Spektrums gelang erst durch Vergleich mit der 4,4-Dideuterio-Verbindung ( $3 b)$. Mit Natriumamid in Benzol ${ }^{[5]}$ erhält man (2b) aus Trideuteriomethyl - triphenylphosphonium - bromid ohne D - Austausch. NMR-Spektrum $\left(\mathrm{CDCl}_{3}\right)$ von $(3 b): \mathrm{H}-3$ als verbreitertes Dublett bei $\tau=5,32$ mit $J_{35}=8,1 \mathrm{~Hz}$.

Die Hydrolyse von (3a) in Dioxan/Wasser $(5: 1 \mathrm{v} / \mathrm{v})$ bei $100{ }^{\circ} \mathrm{C}$ führt mit $81 \%$ Ausbeute zum Diphenyl-(1,2-diphenyläthyl)phosphin-oxid (5), das mit einem authentischen Präparat [6] übereinstimmt. Das daneben erwartete $N$-Phenylhydroxylamin läßt sich als $C, N$-Diphenylnitron isolieren,

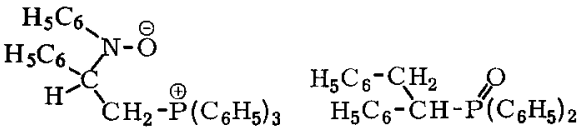

(4)

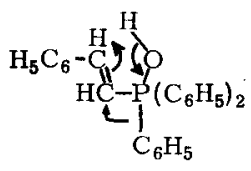

(6)

wenn man (3a) in Gegenwart von Benzaldehyd hydrolysiert. Der zu (5) führende Reaktionsweg - (6) wäre als Zwischenstufe denkbar - ist ähnlich unklar wie die Bildung von (5) aus Methyl-triphenylphosphonium-Salz und Benzaldehyd mit Natriumäthylat in Äthanol [7].

Undurchsichtig ist auch die Thermolyse von (3a) in siedendem Xylol, die unter formalem Verlust von Dehydrobenzol mit $38 \%$ Ausbeute $(7 a)$ ergibt; $\mathrm{Fp}=204-205^{\circ} \mathrm{C}$; IR (KBr): $3320(\mathrm{NH}), 1182 \mathrm{~cm}^{-1}(\mathrm{P}=\mathrm{O})$. Zur unabhängigen Synthese hydrolysiert man das aus (2a) und Benzyliden-anilin in Äther erhaltene Zwitterion (8) und erhält mit $91 \%$ Ausbeute (7a).

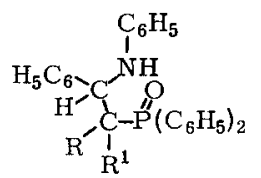

(7)

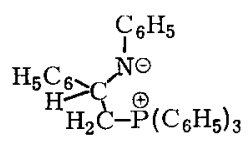

(8)

$$
\begin{aligned}
& \text { (a), } \mathrm{R}=\mathrm{R}^{1}=\mathrm{H} \\
& \text { (c), } \mathrm{R}=\mathrm{C}_{6} \mathrm{H}_{5}, \mathrm{R}^{1}=\mathrm{H} \\
& \text { (d), } \mathrm{R}=\mathrm{R}^{1}=\mathrm{CH}_{3}
\end{aligned}
$$

Die langsamere Umsetzung von Benzyliden-triphenylphosphoran (2c) mit (l) in Benzol ergibt nach $24 \mathrm{Std}$. bei $60^{\circ} \mathrm{C}$ das Cycloaddukt (3c) mit $39 \%$ Ausbeute; Zers.-P. = 146-147 ${ }^{\circ} \mathrm{C}$. NMR $\left(\mathrm{CDCl}_{3}\right): \tau=5,13(\mathrm{H}-3, \mathrm{t})$ mit $\mathrm{J}_{34}=$ $J_{35}=6,5 \mathrm{~Hz}, \tau=5,67(\mathrm{H}-4, q)$ mit $J_{45}=10,0 \mathrm{~Hz}$. Das 31P-Signal $\left(\mathrm{CDCl}_{3}\right)$ liegt bei $+57,9 \mathrm{ppm}$. Die Thermolyse von (3c) in Xylol ergibt mit $24 \%$ Ausbeute das Phosphinoxid (7c), $\mathrm{Fp}=228-229^{\circ} \mathrm{C}$; IR $(\mathrm{KBr}): 3360(\mathrm{NH}), 1178 \mathrm{~cm}^{-1}$ $(\mathrm{P}=\mathrm{O})$.

Auch Isopropyliden-triphenylphosphoran (2d) nimmt (1) in Äther bei $20^{\circ} \mathrm{C}$ auf. Man isoliert mit $22 \%$ Ausbeute das Addukt ( $3 d$ ), Zers.-P. $=142-143^{\circ} \mathrm{C}$, neben $15 \%$ Triphenylphosphin. NMR-Spektrum $\left(\mathrm{CDCl}_{3}\right)$ von $(3 d): \tau=9,09$ (4- $\mathrm{CH}_{3}$, d) mit $\mathbf{J}_{45}=21 \mathrm{~Hz}, \tau=8,37\left(4^{\prime}-\mathrm{CH}_{3}\right)$ mit $\mathbf{J}_{4}{ }^{\prime} 5=$ $18 \mathrm{~Hz}, \tau=5,01(\mathrm{H}-3, \mathrm{~s})$. ${ }^{31} \mathrm{P}-$ Signal $\left(\mathrm{CDCl}_{3}\right):+46,3 \mathrm{ppm}$. Die Hydrolyse von (3d) in siedendem Dioxan/Wasser $(10: 3 \mathrm{v} / \mathrm{v})$ sowie die Pyrolyse im Hochvakuum bei $150^{\circ} \mathrm{C}$ führen zum gleichen Produkt (7d). Das (2-Anilino-1,1-dimethylphenäthyl)diphenylphosphin-oxid schmilzt bei 217 bis $219^{\circ} \mathrm{C}$ und stimmt mit einem aus ( $\left.2 d\right)$ und Benzyliden-anilin nach anschließender Hydrolyse erhaltenen Präparat überein. NMR-Spektrum $\left(\mathrm{CDCl}_{3}\right)$ von $(7 d): \tau=8,69\left(\mathrm{CH}_{3}, \mathrm{~d}\right) \mathrm{mit}$ $\mathrm{J}=14 \mathrm{~Hz}, \tau=8,89\left(\mathrm{CH}_{3}, \mathrm{~d}\right) \mathrm{mit} \mathrm{J}=16 \mathrm{~Hz}, \tau=5,84$ (tert. $\mathrm{H}, \mathrm{d}$ breit) mit $\mathrm{J}=7,0 \mathrm{~Hz}$. IR (KBr): $3320 \mathrm{~cm}^{-1}(\mathrm{NH})$.

$N$-Methyl- $C$-phenylnitron reagiert langsamer als (l) mit Methylen-triphenylphosphoran (2a) in Äther. Das Cyclo-

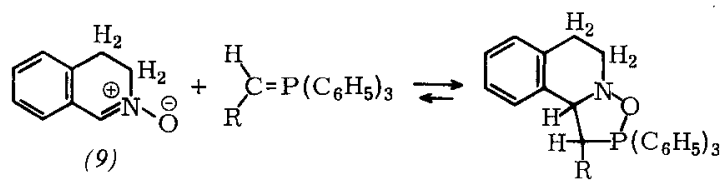

(a), $\mathrm{R}=\mathrm{H}$

(10)

(b), $\mathrm{R}=\mathrm{C}_{6} \mathrm{H}_{5}$ 